\title{
Performance Evaluation of Thin Film Transistors: History, Technology Development and Comparison: A Review
}

\author{
Anchal Sharma \\ Charu Madhu \\ Jatinder Singh \\ Department of Electronics and Communication \\ University Institute of Engineering and Technology, Panjab University \\ Chandigarh, India.
}

\begin{abstract}
In the past several years, the thin film transistor technology has progressed intensely, especially in the low temperature, large-area, high throughput fabrication process. Recently, various thin film transistors (TFT) technological sources have been realized, indicating that new information appliances that match new information infrastructures and lifestyles will be available in the future. In this paper, we review different types of TFTs, including amorphous silicon, polysilicon, organic and oxide TFTs. We report here on different techniques e.g. fabrication and simulation based which has been developed to accurately simulate TFT characteristics and to improve the understanding of the device operation. Here, we highlight the problem gap in the TFT technology that includes downscaling of TFTs and stability of p-type zinc oxide ( $\mathrm{ZnO})$ TFTs and ways to improve it.
\end{abstract}

\section{General Terms}

Thin film transistor structure, fabrication, simulation, mobility.

\section{Keywords}

Thin film transistor (TFT), zinc oxide ( $\mathrm{ZnO})$, amorphous, poly-silicon, organic, and oxide.

\section{INTRODUCTION}

Although the concept of the field effect transistor is patented in 1925 by Julius edger lilienfeld and in 1934 by Osker Heil, but it gets much attention in late 1970s only. Whilst several device structures and semiconductor materials like Te, CdSe, $\mathrm{Ge}$ and $\mathrm{InSb}$ are examined, the competition from the metal oxide semiconductor field effect transistor (MOSFET) based on silicon technology led the thin film transistor (TFT) to backdrop. The requirement of low cost electronics for large area applications like in displays motivates the scientists to think about alternatives for crystalline silicon in the early 1970s. This makes TFT technology a forerunner in this scenario. In 1979, the three scientists Spear, Ghaith and LeComber use hydrated amorphous silicon (a-Si: $\mathrm{H}$ ) as a semiconductor material to describe TFT. Afterwards, technology undergoes several modifications in the use of active channel layer material to achieve significant carrier mobility and switching ratio. Since the mid-1980s, the siliconbased TFTs have successfully influenced the large area liquid crystal displays (LCD) technology and become the most important devices for active matrix liquid crystal and organic light emitting diode applications. For the meantime, TFTs based on organic semiconductor channel layers are introduced in 1990 s with electron mobility equivalent to that of a-Si: H.
This class of TFTs proves to be a potential candidate for incorporation onto flexible substrates. But, they also suffer from reduced carrier mobility which limits its applications in circuits requiring high current and high speed operations. More recently, the TFT concept is fused with transparent semiconducting oxides to create transparent thin-film transistor (TTFT). The paper is ordered as follows: Section II presents different structures of TFT. Section III gives basic operation of TFT. Section IV presents types of TFTs and their performance evaluation and finally conclusion is drawn in section V.

\section{TFT STRUCTURE}

TFT can be configured into four basic structures on the basis of position of the electrodes: top gate top contact, top gate bottom contact, bottom gate bottom contact and bottom gate top contact. It is of great interest that different TFT structures can display quite dissimilar device characteristics while using the exact same materials. In a coplanar configuration, the source drain contacts and the insulator layer are on the same part of the channel whereas, in a staggered configuration, the source drain contacts and the insulator layer are on the opposite part of the channel. Both the staggered and coplanar configurations are further categorized as bottom gate and topgate structures.

Different steps taken for the TFT fabrication typically determines the selection of a particular structure. The deposition of semiconductor channel layer is firstly made in coplanar top gate structure, while deposition of insulator layer is firstly made in the bottom gate structure. The coplanar structure is difficult to realize in a-Si: H TFTs. a-Si: H TFTs use ion implantation technique to form an ohmic contact to the semiconductor.

The channel must be protected during the implantation process to avoid the damage from implantation. The coplanar structure, due to a smaller effective contact area, has increased parasitic resistance as compared to the staggered structure. In addition, staggered structure is commonly used in amorphous silicon TFT, while the coplanar structure is popular in polycrystalline silicon TFT [1].

\section{BASIC OPERATION OF TFT}

A thin film transistor is a special type of field effect transistor formed by placing thin films of the dielectric layer as well as an active semiconductor layer and metallic contacts onto a supporting substrate. TFT differs from MOSFET in certain ways. Firstly, TFT works in accumulation layer while MOSFET works under inversion layer. Secondly, TFT is 


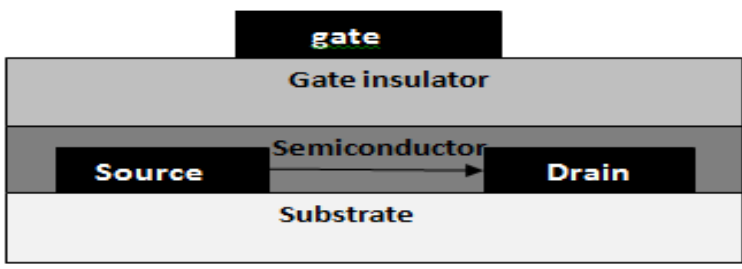

(a)

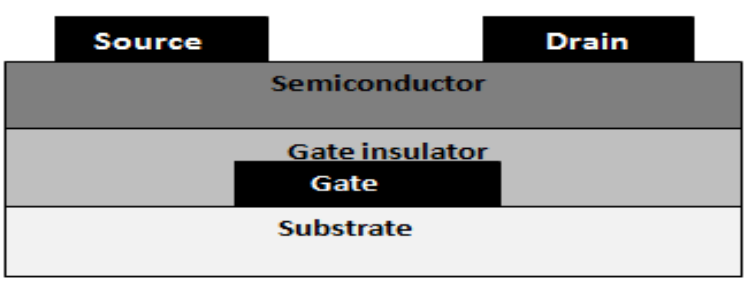

(c)

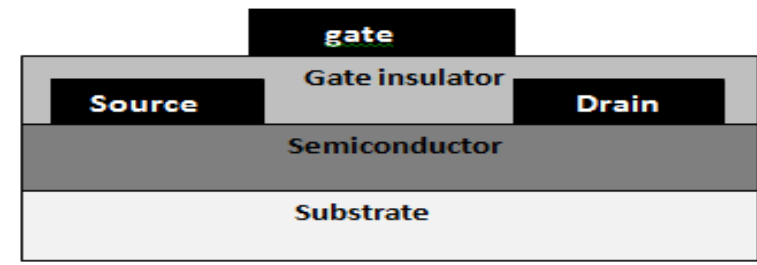

(b)

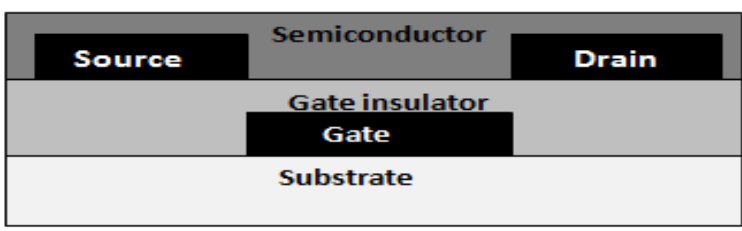

(d)

Fig. 1: TFT configurations: (a) Top-gate bottom-contact and (b) Top-gate top-contact (c) Bottom-gate top-contact; and (d) Bottom-gate bottom-contact. The dashed line indicates charge flow [2].

amorphous in nature as compared to MOSFET, which is crystalline in nature. Thirdly, TFT is undoped while MOSFET is mostly $\mathrm{Si}$-doped. The working principle for TFT is as shown in figure 2 . The applied gate voltage $\left(\mathrm{V}_{\mathrm{G}}\right)$ controls the electrons flow from source to drain. For positive gate voltage, electrons attract towards the bottom side of the semiconductor layer and conduct a channel. Then, the voltage applied between the two terminals result in current flow. The current flow occurs from drain to source.

TFT can be operated in depletion mode or enhancement mode depending on whether it requires a gate voltage to induce the channel conduction. When no gate voltage is applied, the channel conductance is low for enhanced mode of operation. Therefore a low carrier density in the channel is essential to achieve this mode. A channel has to be induced in case of enhancement mode. In this mode, channel enhances on increasing the gate source voltage. Then applying voltage between the drain and source terminals causes the drain current to flow. While in case of depletion mode, if we apply the drain source voltage, the drain current will flow for zero gate source voltage. In this type of MOSFET, there is an implanted channel.

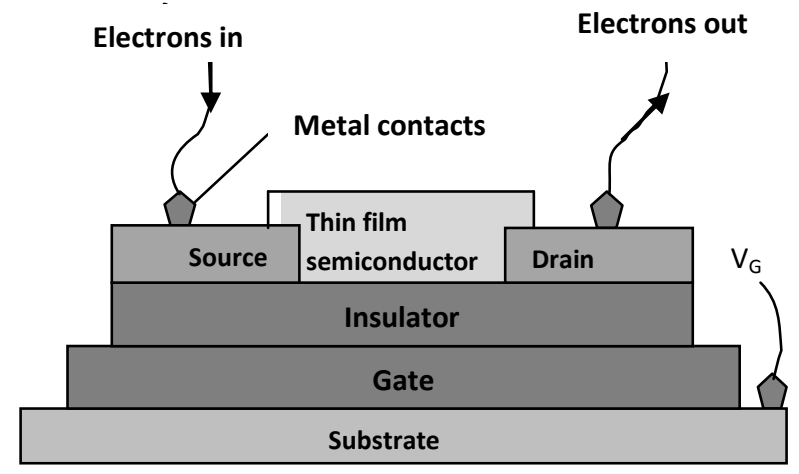

Fig. 2: Basic Thin Film Transistor Structure

\section{TYPES OF TFT \\ 4.1 Amorphous Silicon TFT}

Hydrogenated amorphous silicon (a-Si: H) TFTs are important devices for large scale applications. There are different techniques used for a-Si: H TFTs. These include plasma enhanced chemical vapor deposition (PECVD) and hot wire chemical vapor deposition (HWCVD). The latter one gives high field effect mobility of $4.7 \mathrm{~cm}^{2} / \mathrm{Vs}$ in top gate and $1.5 \mathrm{~cm}^{2} / \mathrm{Vs}$ in bottom gate TFTs [3]. Furthermore hot wire deposited silicon nitride is demonstrated in bottom gate TFTs. Plasma deposited a-Si: $\mathrm{H}$ devices with such gate insulator have field effect mobility of $0.6 \mathrm{~cm}^{2} / \mathrm{Vs}$. In the bottom-gate structure of amorphous silicon with light shield, the sideline is covered with largely phosphorous doped amorphous Si layer. It develops a new technology for manufacturing a-Si: H TFTs of high performance. This helps to reduce the discharging path that exists into the parasitic contacts across the source drain metal and sideline of a-Si: $\mathrm{H}$ island. Hence, this TFT devices exhibit better carrier mobility due to the extensive improvement in resistance between the metal interconnects [4].

\subsection{Poly-silicon TFT}

The growth in polycrystalline silicon thin film transistors (poly-Si TFTs) over the past several years has been restored by the rapid commercialization of flat panel technology including active matrix liquid crystal displays (AMLCDs). A number of ways have been examined for the formation of these TFTs. These include direct deposition methods, such as plasma enhanced and low pressure chemical vapor deposition as well as conversion using amorphous silicon layer. Other techniques include high temperature and low temperature technology. The former is based on the use of quartz substrate, while the latter is based on the use of glass substrate [5]. The principle method of deposition is low pressure chemical vapor deposition (LPCVD) making use of silane [6]. Disilane can also be used in place of silane because of its higher settling rate [7].

Directly deposited poly-Si material with columnar structure deposited at a reduced pressure can yield higher field effect mobility and fewer intragrain defects [8]. There are details regarding plasma enhanced deposition using silane and 
tetraflourosilane gas combination or hot wire 'catalytic' deposition [9]. These techniques will be of great importance, if can be revealed over large areas.

The performance of double gate polysilicon TFTs with n-type channel has been studied using a two dimensional device simulation tool. The simulations are performed based on the properties of the device which are obtained from fabrication of single gate devices. By testing single gate TFTs for varying channel lengths, a particular set of density of states is obtained. These results conclude that double gate TFTs exhibit superior performance in terms of sub threshold slope, driving current and device stability as compare to their single gate TFTs due to their increased gate controllability [10].

\subsubsection{Comparison between amorphous and polysilicon TFTs}

The a-Si: H TFT has $\mu_{\text {eff }}<1 \mathrm{~cm}^{2} / \mathrm{Vs}$, an on off ratio $\left(\mathrm{I}_{\mathrm{on}} / \mathrm{I}_{\text {off }}\right)$ $>10^{6}$, an off current $\left(\mathrm{I}_{\text {off }}\right)$ of $<10-12$ Amp, a threshold voltage $\left(\mathrm{V}_{\mathrm{t}}\right)$ of $<3 \mathrm{~V}$ and a sub threshold slope $(\mathrm{S})$ of $<0.5 \mathrm{~V} / \mathrm{dec}$. But, there are few shortcomings in the a-Si: $\mathrm{H}$ device. Firstly, the mobility is too low for large current or high-speed applications, such as the driving circuit in the organic light emitting diode (OLED) display. Secondly, being is a photoconductor; these TFTs have a large leakage current when expose to light. This problem is solved by using an opaque material to cover the TFT [11]. Thirdly, the stability of the TFT, i.e., threshold voltage shift under immense stress conditions [12].

The intrinsic nature of the chemical bonding fundamentally limits the carrier mobility in the amorphous semiconductors like a-Si: $\mathrm{H}$. These materials show lower carrier as compare to its crystalline counterpart [1]. Moreover, the poly Silicon TFTs shows dominant performance by energy distribution of trapping states at the grain boundaries. However, amorphous semiconductors are favored over polysilicon for channel layer from the perspective of stability of device characteristics and processing temperature [2].

Table 1: Comparison between amorphous and polysilicon TFT

\begin{tabular}{|l|l|l|}
\hline Properties & $\begin{array}{l}\text { Amorphous } \\
\text { silicon TFT }\end{array}$ & Poly silicon TFT \\
\hline Base material & $\begin{array}{l}\text { Amorphous } \\
\text { silicon (Si) }\end{array}$ & Polycrystalline Si \\
\hline $\begin{array}{l}\text { Process } \\
\text { Temperature }\end{array}$ & $300^{\circ} \mathrm{C}$ & $1000^{\circ} \mathrm{C}$ \\
\hline $\begin{array}{l}\text { Mobility } \\
\left(\mathrm{cm}^{2} / \text { Vs }\right)\end{array}$ & Less than 1 [8] & $20-500[9]$ \\
\hline $\begin{array}{l}\text { Structure used } \\
{[10]}\end{array}$ & Staggered & Coplanar \\
\hline $\begin{array}{l}\text { Frequency } \\
(\text { Hz) }\end{array}$ & $100 \mathrm{~K}$ & $10 \mathrm{M}$ [11] \\
\hline Application & $\begin{array}{l}\text { Laptop screen, PC } \\
\text { monitor, LCD TV }\end{array}$ & $\begin{array}{l}\text { Projection light } \\
\text { valves, viewfinders, } \\
\text { laptop screens }\end{array}$ \\
\hline
\end{tabular}

\subsection{Organic TFT}

Organic thin film transistors (OTFTs) are drawing much attention because of their future applications to flexible displays, smart cards and ID tags [13-15]. The performance has been improved to a great extent in recent years. The field effect mobility in the case of pentacene TFTs using $\mathrm{Al}_{2} \mathrm{O}_{3}$ gates on Si substrates has been reported to be $5 \mathrm{~cm}^{2} / \mathrm{Vs}$ [16]. In 1984, the first organic transistor is made using an electrolyte as the gate medium [17]. In 1986, the first organic field-effect transistor is reported by Tsumura et al. that demonstrates clear transistor behavior [18]. OTFTs are of great interest compare to the conventional silicon technology due to their less complex fabrication process. In addition to this, organic semiconductors are well suited with variable plastic substrates at low temperatures, hence can be placed on the substrates at such temperatures $[19,20]$. An OTFT is parallel to inorganic TFT in basic function and design. Typical value of the mobility parameter gives $<1 \mathrm{~cm}^{2} / \mathrm{Vs}$ for amorphous silicon devices as compare to $<10 \mathrm{~cm}^{2} / \mathrm{Vs}$ mobility for organic materials [21, 22]. In addition, the on/off ratio gives the switching performance of OTFTs as high as $10^{6}[23,24]$. Top gate devices have been studied to give better results as compare to the bottom gate devices because of reduced contact resistance [25]. However, there exist a number of challenges in this area which include stability of OTFT in the air, alignment among organic molecules and large driving voltage.

Although there is a lot of research relating OTFT characteristics and its structure, but the conclusions are complex and diverse in nature [26]-[29]. That is due to the issue to differentiate between the structure and the process effects. However, the structure dependence of OTFT characteristics is studied using $2 \mathrm{D}$ simulation. It explains that staggered structure OTFT has better electrical properties than coplanar OTFT [30]. Considering schottky barrier contact, the staggered structure OTFT has higher current flow, lower contact resistance and higher field effect mobility than the planar structure. A schottky barrier is a potential energy barrier for electrons formed at a metal-semiconductor junction.

In a recent study, the structure-dependent contact barrier effects of staggered and inverted coplanar bottom contact structures for organic thin film transistors have been compared on the basis of their electrical performance and further investigated by numerical simulations [31]. It has been concluded that the inverted coplanar devices are more tolerant to the contact barrier effects. These findings can prove helpful in practical circuit integration applications in terms of process development and device structure for organic TFTs.

\subsection{Oxide TFT}

An oxide TFT is a special type of field effect transistor formed by placing thin films of a dielectric and semiconductor active layer and metallic contacts onto a suitable substrate. While amorphous silicon TFT uses amorphous silicon as the material of the electron channels, oxide TFT uses oxide material. Amorphous and crystalline metal oxide TFTs has attracted much attention in recent years. Heavily doped c-Si wafers with oxide thickness of $150 \mathrm{~nm}$ have been used for transistors with $\mathrm{SiO}_{2}$ gate insulator. While, in case of hot wire deposition of silicon, a hydrogen analysis of the oxide is done [3]. This treatment improves the bond between the oxide and the silicon film and increases the field effect mobility. 
Among several materials used in amorphous oxide semiconductors (AOSs) [32, 33], amorphous InGaZnO has drawn much attention as a favorable channel material. These TFTs because of their enhanced performance in terms of mobility, stability, spatial uniformity and transparency to visible light, find importance in other electronic devices. Moreover, the model of the a-InGaZnO TFTs is simpler than a-Si: H TFTs [34]. In another study, different gate insulators like $\mathrm{SiO}_{\mathrm{X}}, \mathrm{SiN}_{\mathrm{X}}$ and $\mathrm{SiO}_{\mathrm{X}} / \mathrm{SiN}_{\mathrm{X}}$ are measured by modeling of amorphous InGaZnO. This helps to investigate the correlation between subgap density of states (DOS), channel resistance and field effect mobility. Due to comparatively large channel resistance values, high subgap DOS levels give low field effect mobility values and vice versa [35].

Recently, $\mathrm{ZnO}$ has developed as a substitute material for TFTs under wide area operations. It is a wide bandgap semiconductor of II-VI semiconductor group ( 3.3-3.4 eV), which possesses high electron mobility, efficient photon emission and can be easily n-doped. ZnO TFTs have a higher mobility value than Si-based TFTs for the same degree of crystallanity. We can fabricate transparent oxide TFTs by using transparent electrodes and semiconductors such as indium tin oxide (ITO). But, there is global shortage of indium which put $\mathrm{ZnO}$ under consideration. $\mathrm{ZnO}$ as a semiconductor material gives low resistance and high transparency as compare to ITO. ZnO is quite appealing as a transparent contact layer due to its high translucency and conductivity, relatively low deposition temperature, low cost and non-toxicity [36]. Moreover, a study of the characteristics of bottom gate enhancement mode TFTs based on zinc oxide $(\mathrm{ZnO})$ as an active channel layer deposited by two different methods, example, radio magnetron sputtering and sol-gel technique at room temperature is reported [37]. Enhancement mode TFTs with sol-gel derived $\mathrm{ZnO}$ channels are found to show better field effect mobility as compared to RF-sputtered TFTs. In addition to this, the simulation tool can be used to optimize predicted performance of RF sputtered and sol-gel TFT for specific applications.

On the other hand, GaN based TFTs attracted much attention in research field in areas of high speed, high power and high processing temperature due to better transport properties of 2D electron gas channel [38]. One of the areas of research in this field is to place high quality GaN thin films using low cost substrate under low temperature. Moreover, low electrical stability of $\mathrm{ZnO}$ TFTs is also affecting its commercialization [39]. As a result, top gate and bottom gate n-type GaN TFTs with good electrical performance and low processing temperature are fabricated $[40,41]$. These $\mathrm{GaN}$ TFTs play significant role in future generation flat panel displays.

The rapid revolution in electronics design provides more opportunities and demands for performance improvement of TFTs and thus downscaling of TFTs becomes more and more critical. The challenges for continuous scaling of TFTs are self-heating effects and short channel effects [42]. The quest for stable $\mathrm{p}$-type $\mathrm{ZnO}$ is challenging because the optical and electronic properties of $\mathrm{ZnO}$ are very susceptible to minute concentration of dopants, impurities and minute perturbation of the lattice [43]. The challenges behind p-type doping of $\mathrm{ZnO}$ is issue of low doping solubility, fabrication of low-onvoltage light emitting diode, laser diode and p-n junction detectors .

\section{CONCLUSION}

In the past several years, the thin film transistor technology has progressed intensely, especially in the low temperature, large-area, high throughput fabrication process. To date, different types of TFTs with the improved mobility, novel structures or flexible material properties have been reported for applications in displays, sensors, imagers, detectors, flexible electronics, etc. We report here on different techniques e.g. fabrication and simulation based which has been developed to accurately simulate TFT characteristics and to improve the understanding of the device operation. The simulation works remarkably help in describing the composite device events. The uniqueness of the thin film transistor technology lies in the composing materials and versatile structure with few limits on the substrate material and size. Oxide and organic TFTs have been studied as potential substitutes for Si-based technology for improving carrier mobility and lowering production costs.

\section{ACKNOWLEDGEMENTS}

The authors would like to thank the anonymous reviewers for their useful comments.

\section{REFERENCES}

[1] Saji, k., J., 2011. Experimental Techniques, Characterization Tools and Thin Film Transistors. pp.105-126.

[2] Lars, H., 2011. Electrolyte-Gated Organic Thin Film Transistors. Linköping Studies in Science and Technology. Dissertations, pp. 1389.

[3] Stannowski, B., October 1971. Silicon-based thinfilm transistors with a high stability. Dissertation, pp. 61-63

[4] Chi-Wen Chen, Ting-Chang Chang, Po-Tsun Liu, Hau-Yan Lu, Kao-Cheng Wang, Chen- Shuo Huang et al., Oct 2005. High-Performance Hydrogenated Amorphous-Si TFT for AMLCD and AMOLED Applications. IEEE electron device letters, vol. 26, pp. 10.

[5] Brotherton, S., D., February 1995. Polycrystalline silicon thin film transistors. Semiconductor Science Technology, vol.10, pp .721-738.

[6] Adams A C, VLSI Technology, ed., S M Sze, 1983, New York McGraw Hill, pp. 93.

[7] Nakazawa K, 1991, J. App. Phys., vol. 69, pp. 1703.

[8] Meakin D B, Coxon P A, Migliorato P, Stoemenos J, and Economou N A, 1987, Applied Physics Letters, vol. 50 , pp.1894.

[9] Matsumura H, Hosoda Y and furukawa S, 1993, Mater, Res. Soc. Symp. Proc., vol.283, pp. 623.

[10] I. Pappas, D. Tassis, s. Siskos, and C. A. Dimitriadis, Jun 2010. Characteristics of DoubleGate Polycrystalline Silicon Thin-Film Transistors for AMOLED Pixel Design. IEEE, vol.978, pp. $4244-8157$.

[11] Kuo, Y., 2013. Thin Film Transistor Technology Past, Present, and Future. The Electrochemical Society Interface, spring.

[12] Powell, M., J., Berkel, C., Franklin, A., R., Deane, S., C., and Milne, W., I., February 1992. Defect 
pool in amorphous-silicon thin-film transistors. Phys. Rev. B, Condens. Matter, vol. 45, pp. 41604170.

[13] A. Lodha, and R. Singh, 2001, IEEE Transaction Semiconductor Manufact., vol. 14, pp. 281.

[14] Song C., K., and Y. X. Xu, 2003, J. Korean Physics Society, vol. 42, pp. 425.

[15] S. H. Jin, J. S. Yu, Lee, C., A., Kim, J., W., B. G. Park, and J. D. Lee, 2004, J. Korean Physics Society, vol.44, pp.181.

[16] Kelley, T., W., Muyres, D., V., Baude, P., F., Smith, T., P., and Jones, T., D., 2003, Materials Research Society Symposium Proceeding, San Francisco, pp. 226.

[17] White, H., S., Kittlesen, G., P., and Wrighton, M., S., 1984, J. Am. Chem. Society, vol.106, pp. 5375.

[18] Tsumura, A., Koezuka, H., and Ando, T., 1986, Applied Physics Letter, vol. 49, pp. 1210.

[19] Bao, Z., Rogers, J. A., Katz, H. E., 1999, J. Mater. Chem., vol. 9, pp. 1895.

[20] Rogers, J. A., Bao, Z., Katz, H. E., Dodabalapur, 2003, A. - Thin-Film Transistors, volume-377.

[21] Kelley, T. W., et al., 2003, Mater. Res. Soc. Symp. Proc., vol. 771, pp. 169.

[22] Sundar, V., C., Zaumseil, J., Podzorov, V., Menard, E., Willett, R., L., Someya, T., et al., 2004, www.sciencemag.org, vol. 303, pp. 1644.

[23] Dimitrakopoulos, C. D., and Malenfant, P. R. L., 2002, Adv. Mater., vol. 14, pp. 99.

[24] Kagan, C. R., 2003, Thin Film Transistors, Marcel Dekker Inc., New York.

[25] Roichman, Y., and Tessler, N., 2002, Appl. Phys. Lett., vol. 80, pp. 151.

[26] Necliudov, P., V., Shur, M., S., Gundlach, D., J., and Jackson, T., N., Dec. 2000. Modeling of organic thin film transistors of different designs. J. Appl. Phys., vol. 88, pp. 6594-6597.

[27] Roichman, Y., and Tessler, N., Jan. 2002. Structures of polymer field-effect transistor: Experimental and numerical analyses. Appl. Phys. Lett., vol. 80, pp. 151-153.

[28] Hill, I., G., Oct. 2005. Numerical simulations of contact resistance in organic thin-film transistors. Appl. Phys. Lett., vol. 87, pp. 163 -505.

[29] Gundlach, D., J., Zhou, L., Nichols, J., A., Jackson, T., N., Necliudov, P., V., and Shur, M., S., Jul. 2006. An experimental study of contact effects in organic thin film transistors. J. Appl. Phys., vol. 100, pp. 024- 509.

[30] Chang-Hoon Shim, Fumito Maruoka, and Reiji Hattori, Jan 2010. Structural Analysis on Organic Thin-Film Transistor with Device Simulation. IEEE transactions on electronic devices, vol. 57, No. 1.

[31] Linrun Feng, Xiaoli $\mathrm{Xu}$, and Xiaojun Guo, December 2012. Structure-Dependent Contact Barrier Effects in Bottom-Contact Organic Thin-
Film Transistors. IEEE transactions on electronic devices, vol. 59, No. 12.

[32] H. Hosono, M. Yasukawa, and H. Kawazoe, Aug. 1996. Novel oxide amorphous semiconductors: Transparent conducting amorphous oxides. J. NonCryst. Solids, vol. 204, pp. 334-344.

[33] Hosono, H., Jun. 2006. Ionic amorphous oxide semiconductors: Material design, carrier transport, and device application. J. Non-Cryst. Solids, vol. 352, pp. 851-858.

[34] Abe, K., Kaji, N., Kumomi, H., Nomura, K., Kamiya, T., Hirano, M., et al., October 2011. Simple Analytical Model of on operation of amorphous In- $\mathrm{Ga}-\mathrm{Zn}-\mathrm{O}$ Thin-Film Transistors. IEEE transaction on electron Devices Vol. 58, no. 10 .

[35] Park, S., Cho, E., N., and Yun, I., April 2012. Investigation on the relationship between channel resistance and sungap density of states of amorphous InGaZnO thin film transistors. Solid State Electronics, vol. 75, pp. 93-96.

[36] Umit Ozgur, Daniel Hofstetter, and Hadis Morko, July 2010. ZnO Devices and Applications: A Review of Current Status and Future Prospects. IEEE, vol. 98, pp. 7.

[37] Singh, S., and Chakrabarti, P., 2013. theoretical and experimental studies of characteristics of $\mathrm{ZnO}$ TFTs. Journal of electron devices, Vol. 18, pp. 1543-1548.

[38] H. F. Sun, A. R. Alt, H. Benedickter, E. Feltin, J. F. Carlin, M. Gonschorek, et al., Apr. 2010. 100-nmgate $(\mathrm{Al}, \mathrm{In}) \mathrm{N} / \mathrm{GaN}$ HEMTs grown on $\mathrm{SiC}$ with $\mathrm{FT}$ $=144 \mathrm{GHz}$. IEEE Electron Device Lett., vol. 31, pp. 293-295.

[39] Park, J., S., Jeong, J., K., Chung, H., J., Mo, Y., G., and Kim, H., D., Feb.2008. Electronic transport properties of amorphous indium-gallium-zinc oxide semiconductor upon exposure to water. Appl. Phys. Lett., vol. 92, pp. 072-104.

[40] Rongsheng Chen, Wei Zhou, Meng Zhang, and Hoi Sing Kwok, september 2012. Top-Gate GaN ThinFilm Transistors Based on AlN/GaN Heterostructures. IEEE electron device letters, vol. 33, pp. 9.

[41] Rongsheng Chen, Wei Zhou, Meng Zhang, and Hoi Sing Kwok, April 2013. Bottom Gate Thin-Film Transistors Based on GaN Active Channel Layer. IEEE electron device letters, vol. 34, pp. 4.

[42] Guoa, X., Sporeab, R., Shannonb, J., M., and Silvab, S., R., 2009. Down-scaling of Thin-Film Transistors: Opportunities and Design Challenges. The Electrochemical Society, vol. 22, pp. 227.

[43] Rana, S., B., Singh, A., and kaur, N., june 2012. Structural and optoelectronic characterization of prepared and $\mathrm{Sb}$ doped $\mathrm{ZnO}$ nanoparticles. J Mater Sci: mater electron, vol. 24, pp. 44-52. 